\title{
Kinect-based Visual Communication System
}

\author{
Chao Sun ${ }^{1.2}$, Bing-Kun Bao ${ }^{1.2}$, \\ Changsheng $\mathrm{Xu}^{1.2}$ \\ ${ }^{1} \mathrm{NLPR}$, Institute of Automation \\ Chinese Academy of Sciences, Beijing, China \\ ${ }^{2}$ China-Singapore Institute of Digital Media, \\ Singapore \\ \{csun, bkbao,csxu\}@nlpr.ia.ac.cn
}

\author{
Tao Mei \\ Microsoft Research Asia \\ Beijing 100080, China \\ tmei@microsoft.com
}

\begin{abstract}
Nowadays, most existing online instant messaging tools, such as Live Messenger, Google Talk, Yahoo Messenger, ICQ, enable people to communicate with each other no matter where and when they are. However, it is still difficult for people who speak different native languages and do not understand each other's to communicate smoothly. It could be more difficult when people with hearing impairment are trying to use those tools. Moreover, users' hands are usually tied up with keyboard and mouse to keep typing messages. To deal with these disadvantages, we design a Kinect-based Visual Communication System (KVCS), which contains following features: (1) Kinect-based sign language recognition module to make deaf-mute persons be able to chat. (2) Kinectbased expression recognition module to enrich online chatting experiences at the same time.(3) Kinect-based speech recognition module to free users' hands when chatting. (4) Cross-media multi-lingual visualized translation module to enable users to catch the meanings of conversation much easier. Experiments on our system demonstrate that this novel KVCS provides a powerful and efficient communication function and a wonderful user experience.
\end{abstract}

\section{Categories and Subject Descriptors}

H.4 [Information Systems Applications]: Miscellaneous; D.2.8 [Software Engineering]: Metrics-complexity measures, performance measures

\section{General Terms}

Theory

\section{Keywords}

Kinect sensor, online communication, cross-media, crosslanguage

\section{INTRODUCTION}

The growing trend toward globalization and informationization has profoundly changed the communication modes in

Permission to make digital or hard copies of all or part of this work for personal or classroom use is granted without fee provided that copies are not made or distributed for profit or commercial advantage and that copies bear this notice and the full citation on the first page. To copy otherwise, or republish, to post on servers or to redistribute to lists, requires prior specific permission and/or a fee.

ICIMCS'12, September 09-11, 2012, Wuhan, China.

Copyright 2012 ACM 978-1-4503-1600-2/12/09 ...\$15.00. many fields such as international trade, tourism and service industries. Online instant messaging tools, such as Windows Live Messenger, Google Talk and Yahoo Messenger, are becoming more and more popular because of the spontaneity and convenience in use.

However, there exist two challenges for these instant messaging tools: input and language obstacles. Input obstacle means that existing tools require users to type texts. Language obstacle denotes that people speaking different languages can not smoothly communicate with each others by existing instant messaging tools. Communication problems caused by input and language obstacles in existing instant messaging tools are foreseeable, as it is inevitable that sometimes people have to instantly communicate with those who not only be on the other side of the ocean but also may have different accents, native languages, typing difficulties, or even hearing impairment.

Both academic and industrial communities have been making efforts in these two limitations. [14] proposed a Kinectbased technology, which generates avatar for each user to reproduce his facial expressions. This technology could enrich user's input experience against monotonous typing input. By seeking the optimal way to lay out word icons such that the resulting picture best conveys the meaning of the input sentence, Goldberg et al. [4] performed a family of intuitive "ABC" layouts which organize icons in three groups to help communication against language obstacle. Beyond academia, to deal with input obstacle, many instant messaging tools try to directly transfer the raw voice or video information captured from users beyond textual information, like Windows Live Messenger ${ }^{1}$, Yahoo Messenger ${ }^{2}$. But when people need to communicate with those who are deaf and mute or speaking different languages, this will not help. Machine translation has also been applied to handle language obstacle. For example, Tencent $\mathrm{QQ}^{3}$ developed a built-in translation in its international version for multi-language users. However, machine translation does not always interpret correctly and accurately. Cases of miscommunication caused by machine mistranslation are not rare and the state-of-theart techniques are far from satisfying expectations of real applications.

To overcome the input and language obstacles, we design a novel instant communicating system, named Kinect-based Visual Communication System (KVCS). As a widely available and low cost device, Microsoft Kinect sensor can provide colorful image, infrared image, depth map, and audio

\footnotetext{
${ }^{1}$ https://messenger.live.com

${ }^{2}$ http://messenger.yahoo.com

${ }^{3}$ http://www.imqq.com
} 


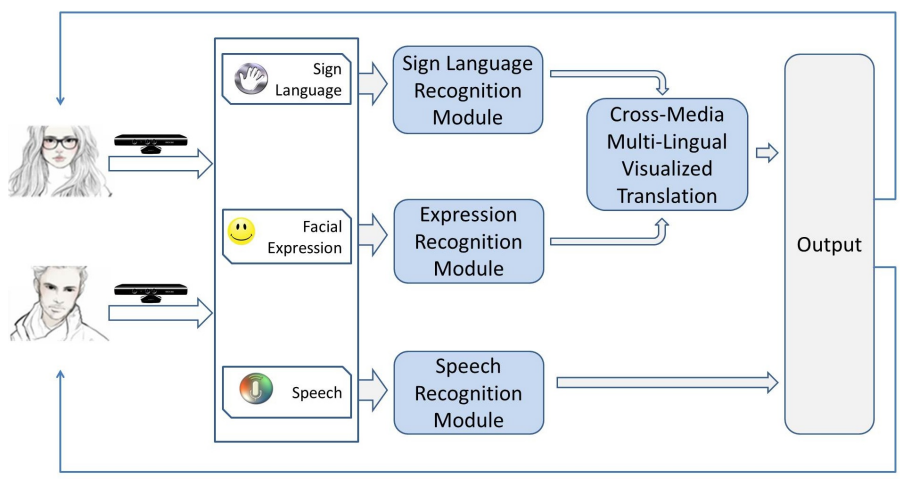

Figure 1: Kinect-based Visual Communication System Framework.

capture function. Hence we select it for our KVCS to tackle the input obstacle. In our KVCS, audio capture is used for speech recognition to free users' hands, color image and depth map are utilized to do sign language and expression recognition to meet deaf-mute users' demand, enrich online chatting experiences. In addition, we also employ text to image conversion technology to tackle the language obstacle, inspired from that visual images could not only transfer the information more accurately and conveniently but also be understood easily and intuitively independent to languages. With this technology, our KVCS can bridge the communication gaps between people with different accents, native languages, or even hearing impairment.

The contributions of our KVCS can be summarized into three-fold:

- We integrate the newly launched technology, Microsoft Kinect sensor, to construct a brand-new communication environment, which is benefit to deaf-mute users, the users with different native languages or accents.

- Compared with those existing messaging tools which use a mouse or keyboard for textual input, our proposed system allows multi-modal inputs, such as sign language, facial expression, voice, which are more natural, intuitive and engaging.

- We utilize text-to-image technology to enable users catch the meanings of conversation much easier.

The rest of the paper is structured as follows: We introduce the framework and appearance of our KVCS in Section 2 and elaborate each module of KVCS in Section 3. Experimental results are reported in Section 4. Finally, we give conclusions in Section 5.

\section{KINECT-BASED VISUAL COMMUNICA- TION SYSTEM OVERVIEW}

We illustrate the principle architecture of the proposed Kinectbased Visual Communication System(KVCS) in Figure 1. User's hand, facial expression and speech are firstly detected and extracted by Microsoft Kinect to input into three modules respectively, that is, Sign Language Recognition, Expression recognition and Speech Recognition modules. The textual content of the conversation as well as expression are then recognized by these three modules. To provide vivid and intuitive visual message corresponding to the conversations, we employ text-to-image converse in Cross-media

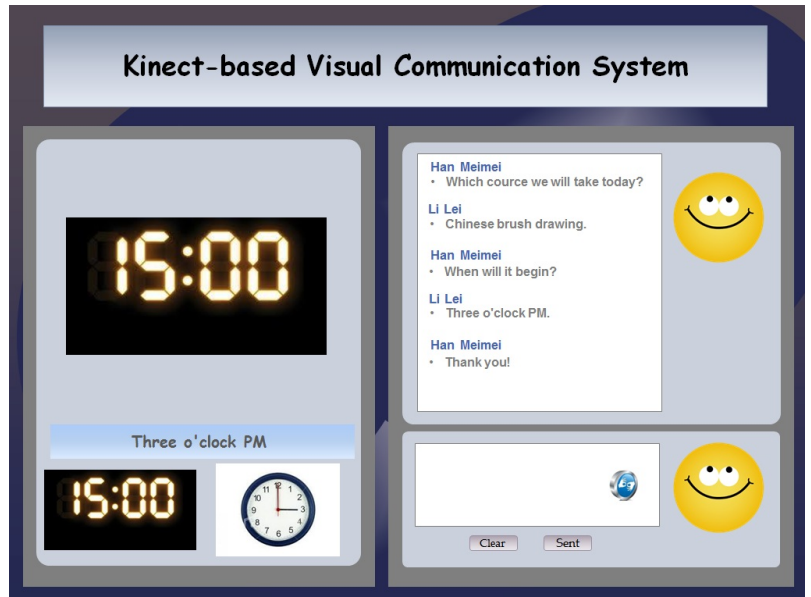

Figure 2: User-Interface of Kinect-based Visual Communication System Framework.

Multi-lingual Visualized Translation Module.

In practice, sign language recognition helps deaf-mute persons to chat, expression recognition enriches online chatting experiences, speech recognition frees users' hands, meanwhile cross-media multi-lingual visualized translation module to enable users to catch the meanings easily. Figure 2 shows the user interface of our Kinect-based visual communication system.

\section{KINECT-BASED VISUAL COMMUNICA- TION SYSTEM}

In this section, we present more details about the major components of the proposed Kinect-based Visual Communication System.

\subsection{Kinect-based Sign Language Recognition}

Nowadays, sign language recognition is a growing research area in the field of gesture recognition. Research has been performed around the world using many different sign languages, including American sign language [2], Chinese Sign Language [3], German sign language [1], and Japanese sign language [9].

A main difficulty in sign language recognition is to capture hand movement by sensor. In computer vision techniques, sensor is typically a camera which can generate depth maps for sign language recognition. Different kinds of sensors are explored varying from tracking systems, like data gloves, to computer vision techniques using camera [11] and motion capture systems [5]. As for now, commercially available depth camera systems were expensive, and only a few researchers have used depth information to recognize hand pose.

Fortunately, the release of Microsoft Kinect sensor has provided a low cost and off-the-shelf choice for depth sensors. The Kinect sensor involves an infra-red(IR) light projector, standard CMOS camera, color camera, and a standard USB interface. The distortion of IR pattern is used to calculate depth maps, which have a per-pixel depth resolution of 1 centimeter while camera is 2 meters far away. The images are $640 \times 480$ and are transferred at 30 frames per second [14]. Those characteristics enable us to recognize sign language using depth data captured from Kinect.

In our KVCS, we focus on American sign language finger- 
spelling, which spells out words by using signs that correspond to the letters of the word. We discard frames where the hand is moving as we are only interested in the static sign. This insures that we do not attempt to classify the transition between two hand shapes.

The recognition technology is followed by Pugeault et al.[8], that is, it includes three steps: (1) Hand extraction and tracking; (2) Hand features extraction; (3) Classification.

Firstly, we use the library ${ }^{4}$ provided by Kinect to detect hands from specified gestures, and track detected hands in $3 \mathrm{D}$ space using the depth image. The hand is then segmented from the depth image. These segmented pixels are then used to form bounding boxed around the hand, both in depth and color camera image. Secondly, we perform Gabor filtering on intensity and depth images to extract hand shape features. Once the hand is detected, both depth and intensity bounding boxes will be convolved with a bank of Gabor filters at 4 scales and 4 orientations. A 2D Gabor filter is typically formed as:

$$
G(x, y, \lambda, \sigma, \theta)=\exp \left(\frac{\tilde{x}^{2}+\tilde{y}^{2}}{2 \sigma^{2}}\right) \exp \left(i\left(\frac{2 \pi x}{\lambda}\right)\right)
$$

where $\tilde{x}=x \cos \theta+y \sin \theta$ and $\tilde{y}=-x \sin \theta+y \cos \theta, \lambda$ is the wavelength, $\sigma$ is the variance of Gaussian envelope and $\theta$ is the orientation. The convolution results are arrays of floating point values of the same size as image, denoted as $I_{1}, I_{2}, \cdots, I_{16}$.

The filtering results are then averaged across overlapping Gaussian basis functions on a $8 \times 8$ grid.

$$
D(i, j, k)=\sum_{x, y} I_{k}(x, y) \exp \left(-\frac{\left(x-x_{i}\right)^{2}+\left(y-y_{i}\right)^{2}}{2 r^{2}}\right)
$$

where $i, j$ are the horizontal and vertical index of the cell, $k$ is the Gabor filter index, and $r=8$ is the width of Gaussian filter. This forms a feature vector for both depth and intensity information.

Finally, the learning and classification are performed using a multi-class random forest. A random forest consists of a population of randomized classification tree $T_{i}$. Given a new sample $v$, a tree will predict the class $T_{i}(v)$ corresponding to the label of the leaf node it falls into. Then the forest yields a confidence that the feature vector $v$ indicates a hand shape $h$ :

$$
P(h)=\frac{1}{N} \sum_{i<N} \delta_{h}\left(T_{i}(v)\right)
$$

where $N$ indicates the number of trees in the forest, $T_{i}(v)$ indicates the leaf of the $i$ th tree $T_{i}$ into which $v$ falls, and $\delta_{h}$ is the Kronecker delta function. This probability distribution is then used to propose likely hand pose candidates.

\subsection{Kinect-based Expression Recognition}

Facial expression is one of the most natural, effective and immediate representations for human beings when communicating with others. Automatic facial expression recognition is an interesting and challenging topic, and could impact important applications in many fields. The original facial expression recognition methods attempt to recognize facial expressions from data collected in a highly controlled environment given high resolution frontal faces[13]. However, in real world applications, the input face images are often at low resolutions. Hence the study on low-resolution facial expression recognition is more important and meaningful[12].

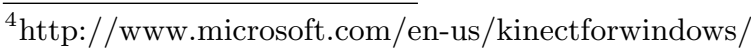

Kinect-base expression recognition module in our KVCS uses Kinect sensor to capture user's facial expression, in lowresolution, and performs expression recognition. Then an avatar representing user's expression and emotion will be shown on KVCS's user interface. This module will not only enrich online chatting experience but also protect user's privacy in special occasions against directly showing user's facial image captured from camera.

We employ the method proposed by Shan et al. in [10]. In KVCS, we perform expression recognition using facial representation based on statistical local features - Local Binary Patterns. The LBP operator labels the pixels of an image by thresholding a $3 \times 3$ neighborhood of each pixel with the center value and considering the results as a binary number, and the 256-bin histogram of the LBP labels computed over a region is used as a texture descriptor. After a image is labeled with the LBP operator, a histogram of the labeled image $f(x, y)$ will defined as:

$$
H_{i}=\sum_{x, y} I((f(x, y)=i)), \quad i=0,1, \cdots, n-1
$$

where $n$ is the number of different labels produced by the LBP operator and

$$
I(A)= \begin{cases}1 & \mathrm{~A} \text { is ture } \\ 0 & \mathrm{~A} \text { is false }\end{cases}
$$

This LBP histogram contains information about the distribution of the local micro-patterns over the whole image, so it can be used to statistically describe image characteristics. However, a LBP histogram does not involve any indication about micro-patterns. Therefore, to add shape information of faces, face images were equally divided into small regions $R_{0}, R_{1}, \cdots, R_{m}$ to extract LBP histograms. Then the concatenated spatially enhanced feature histogram is defined as:

$$
H_{i, j}=\sum_{x, y} I((f(x, y)=i)) I\left((x, y) \in R_{j}\right)
$$

where $i=0,1, \cdots, n-1, j=0,1, \cdots, m-1$. The extracted feature histogram represents the local texture and global shape of face images.

After obtained feature histogram, we use template matching to perform expression recognition. A template is formed for each class of face images, then a nearest-neighbor classifier is used to match the input image with the closest template. The region-based weighted $\chi^{2}$ square statistic is then selected as the dissimilarity measure for histograms:

$$
\chi_{\omega}^{2}(S, M)=\sum_{i, j} \omega_{j} \frac{\left(S_{i, j}-M_{i, j}\right)^{2}}{S_{i, j}+M_{i, j}}
$$

where $S$ and $M$ are two LBP histograms and $\omega_{j}$ is the weight for $j$ th region.

\subsection{Kinect-based Speech Recognition}

Through decades of development, speech recognition, also referred to automatic speech recognition (ASR), has achieved remarkable performance and the word error rates (WER) on some tasks are less than $1 \%$. Speech recognition has been a practical and mature technology, and has been applied to various applications like communication, health care, and military.

Based on the mature speech recognition technology, our KVCS uses Kinect sensor's four-element linear microphone array, which uses 24-bit ADC and provides local signal pro- 
cessing, including acoustic echo cancellation and noise suppression, to capture user's speech. Then it will perform recognition, and translate the voice information to textual information which will afterwards be used by cross-media multi-lingual visualized translation module. This Kinectbased speech recognition module free users from painstakingly typing on keyboards and enables communications between illiterate users.

\subsection{Cross-media Multi-lingual Visualized Trans- lation}

Cross-media multi-lingual visualized translation module is the core module which bridges the gaps between different languages. Although Kinect-based sign language recognition, Kinect-based speech recognition, or Kinect-based expression recognition, are both perform a role of capturing input information from users and transferring these information into textual content, they do not directly tackle the language obstacle. Cross-media multi-lingual visualized translation module is exactly the one which deals with language obstacle, by transferring the textual content into visual image content which can be intuitively understood by users using any languages.

Some closely related work like text-to-image conversion has been done. Zhu et al. [15] proposed a text-to-image system that synthesizes a picture from general, unrestricted natural language text. They identified informative text units, searched the most likely image parts conditioned on text, and optimize the picture layout conditioned on both text and image parts. [7] presented a story picture engine which uses an unsupervised approach to automatically picture story. This engine extracts keywords from story and searched annotated images from a pre-founded database, then arrangs those images using a ranking method.

Our cross-media multi-lingual visualized translation module learns from their methods' strengths. It comprises of four main components: 1)image collection and index; 2)dialogue analysis; 3)image selection; 4)visual layout.

Firstly, in order to meet the real-time requirement, an image database facilitating the communicating visualization should be well collected and indexed in advance. Therefore, we collected a large-scale image set from web(e.g.,Flickr, Google image) attached by tags and built a cluster-based index for keywords by considering the visual and tag information simultaneously. Intuitively, it is hard to find proper images to represent some kinds of semantic concepts, such as "small", "large", as they are nonrepresentational. Hence, after data collection, the image database will be divided into two sub-databases, one corresponds to those words which can be represented by images, other one corresponds to those words which cannot be represented by images. For the first one, we automatically label images using their attached tags. For the second one, limited tags, such as some verbs, adjectives, fixed phrases and interrogatives etc., are considered and labeled to images manually. This step was done offline.

Secondly, Once users start a dialogue, KVCS will identify meaningful keywords and analyze grammatical and logical relationships between these keywords using probabilistic natural language processing to avoid semantic ambiguity. Meaningful keywords which stand for users's intent are used as query words in image selection, while grammatical and logical relationships are used for visual layout.

Thirdly, after detected keywords of dialogue, a two-step approach is applied to select the representative images of key-

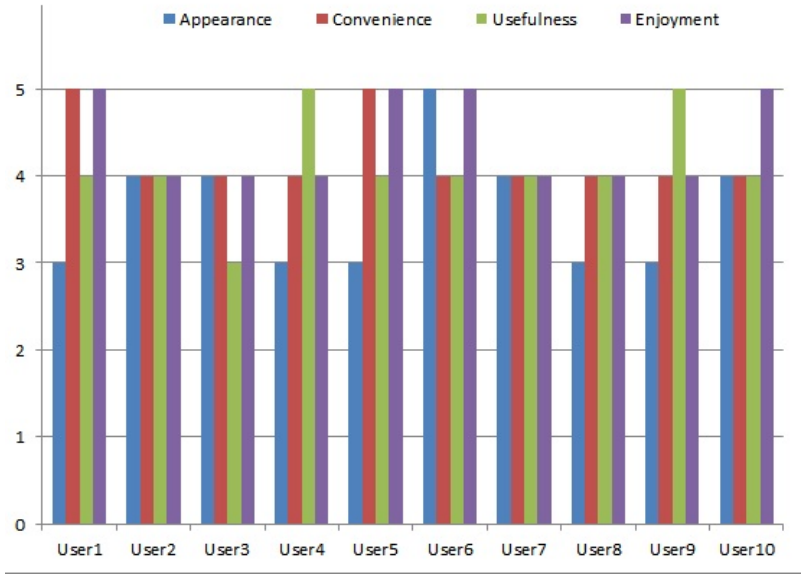

Figure 3: User Study Result on KVCS.

words from the pre-founded image database. In the first step, the most proper sub-cluster is selected from all subclusters pre-founded in image collection and index step, according to context clues. In the second step, by using visual features and tags information, the most fitted images corresponding to keywords are picked up as representative images.

Finally, in order to deliver the meaning of chat sentences, KVCS performs a visual layout with well-designed templates to generate the visual message from those images which correspond to keywords separately and represent only certain concepts but not logical meaning. In the end, the visual messages consists of images sequence are presented to users. More details of this module are introduced in [6].

\section{EXPERIMENTS}

In this section, we first conduct experiment to evaluate the performance on our Kinect-based visual communication system, then give a discussion.

To evaluate the performance, we invited 10 volunteers to test our KVCS. The KVCS includes 15 pre-defined scenes, which are shown in Table 1.

Table 1: All Pre-defined Scenes in KVCS

\begin{tabular}{lll}
\hline & Scenes Topic & \\
\hline ask way & book hotel & borrow book \\
buy air ticket & buy car & buy clothes \\
buy phone & electrical repair & change money \\
movie & order pizza & rent house \\
reserve sickness & select course & travelling \\
\hline
\end{tabular}

Our experiment covered all scenes. The evaluation includes two parts:

Firstly, every volunteer was asked to use KVCS to communicate with others in all 15 pre-defined scenes. Local users were asked to use English, while remote users used Chinese. Then each person gave a score to KVCS on four aspects: appearance, convenience, usefulness, and enjoyment. The score is from 1 to 5 with interval 1, which means 'very bad', 'bad', 'average', 'good', 'very good', respectively. The result is shown on Figure 3. We can see that most of users are satisfied with KVCS. Secondly, every volunteer was required to use KVCS comparing with an instant messaging tool-Windows Live Messenger. Then each person should 


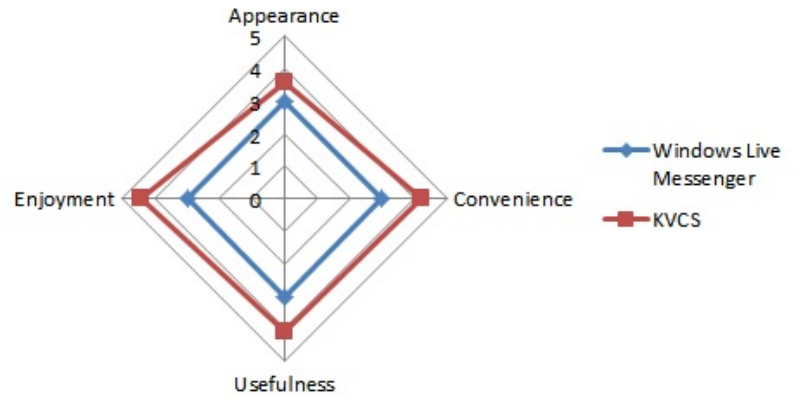

Figure 4: The User Experience Comparison Between KVCS and Windows Live Messenger.

give a score to both communication systems on four aspects: appearance, convenience, usefulness, and enjoyment. The score is from 1 to 5 with interval 1 , which indicates 'much worse', 'worse', 'same', 'better', 'much better', respectively. The result is shown on Figure 4. It is observed that, overall, our KVCS outperforms Windows Live Messenger. From experiments, we can observe that our KVCS can provide a powerful and efficient communication function and a wonderful user experience, as well as outperform other instant messaging tools. The possible explanations include: (1) KVCS has integrated sign language recognition, expression recognition and speech recognition. This kind of input mode is far more interesting than typing, and can strongly improve users' experience. (2) The build-in cross-media multi-language visualized translation module enables persons who have different languages to chat online, and makes this type of communication become easy and convenient.

\section{CONCLUSIONS}

In this paper, we presented a novel online communication system, named Kinect-based Visual Communication System. By employing Microsoft Kinect sensor as the integral and intelligent interface to receive and partially process the multi-modal inputs generated from users, our KVCS first implements sign language recognition, expression recognition and speech recognition, then performs cross-media multilingual visualized translation to assist users' communication. Our system is beyond a simple combination of the translation, sign language recognition, expression recognition and speech recognition technologies. It targets to solve more difficulties facing by current communication systems. Through our KVCS, people who have different languages or even are deaf-mute can communicate smoothly and easily.

\section{ACKNOWLEDGEMENT}

This work is supported in part by the National Natural Science Foundation of China (Grant No. 61003161), the China Post-Doctoral Science Foundation (Grant No. 2011M500430), and Microsoft Research Asia UR Project.

\section{REFERENCES}

[1] B. Bauer, H. Hienz, and K. Kraiss. Video-based continuous sign language recognition using statistical methods. In Proceedings of 15th International Conference on Pattern Recognition, 2:463-466, September 2000.
[2] H. Brashear, T. Starner, P. Lukowicz, and H. Junker. Using multiple sensors for mobile sign language recognition. In Proceedings of the Seventh IEEE International Symposium on Wearable Computers, pages $45-52,2003$.

[3] G. Fang, W. Gao, and D. Zhao. Large vocabulary sign language recognition based on hierarchical decision trees. In International Conference on Multimodal Interfaces, pages 125-131, November 2003.

[4] A. B. Goldberg, X. Zhu, C. R. Dyer, M. Eldawy, and L. Heng. Easy as abc?: facilitating pictorial communication via semantically enhanced layout. In Proceedings of the Twelfth Conference on Computational Natural Language Learning, pages 119-126, 2008.

[5] J. L. Hernandez-Rebollar, N. Kyriakopoulos, and R. W. Lindeman. A new instrumented approach for translating american sign language into sound and text. In Proceedings of the Sixth IEEE International Conference on Automatic Face and Gesture Recognition, pages 547-552, 2004.

[6] Y. Jiang, J. Liu, Z. Li, C. Xu, and H. Lu. Chat with illustration: A chat system with visual aids. 4 th Ingernational Conference on Internet Multimedia Computing and Service, 2012.

[7] D. Joshi, J. Wang, and J. Li. The story picturing engine - a system for automatic text illustration. $A C M$ Trans. on Multimedia Computing, Communications, and Applications, 2:68-69, 2006.

[8] N. Pugeault and R. Bowden. Spelling it out: Real-time asl fingerspelling recognition. In Proceedings of 2011 IEEE International Conference on Computer Vision Workshops, pages 1114-1119, November 2011.

[9] H. Sagawa and M. Takeuchi. A method for recognizing a sequence of sign language words represented in a japanese sign language sentence. In Proceedings of the 4th IEEE International Conf. on Automatic Face and Gesture Recognition, pages 434-439, March 2000.

[10] C. Shan, S. Gong, and P. W. McOwan. Facial expression recognition based on local binary patterns: A comprehensive study. Journal Image and Vision Computing, 27:803-816, May 2009.

[11] T. Starner and A. Pentland. Visual recognition of american sign language using hidden markov models. In Proceedings of the International Workshop on Automatic Face and Gesture Recognition, 1995.

[12] Y. Tian, L. Brown, A. Hampapur, S. Pankanti, A. Senior, and R. Bolle. Real world real-time automatic recognition of facial expression. In IEEE Workshop on Performance Evaluation of Tracking and Surveillance, 2003.

[13] Y.Tian. Evaluation of face resolution for expression analysis. In CVPR Workshop on Face Processing in Video, 2004.

[14] Z. Y. Zhang. Microsoft kinect sensor and its effect. Multimedia, IEEE, 19:4-10, February 2012.

[15] X. Zhu, A. Goldberg, M. Eldawy, C. Dyer, and B. Strock. A text-to-picture synthesis system for augmenting communication. In Proceedings of 22nd national conference on Artificial Intelligence, pages 1590-1595, 2007. 